\title{
Translational gene expression control in Chlamydia trachomatis
}

\author{
Nicole A. Grieshaber, Travis J. Chiarelli, Cody R. Appa, Grace Neiswanger, \\ Kristina Peretti, Scott S. Grieshaber $\mathbb{B}^{*}$ \\ Department of Biological Sciences, University of Idaho, Moscow, ID, United States of America \\ *scottg@uidaho.edu
}

\section{Abstract}

The human pathogen Chlamydia trachomatis proceeds through a multi phenotypic developmental cycle with each cell form specialized for different roles in pathogenesis. Understanding the mechanisms regulating this complex cycle has historically been hampered by limited genetic tools. In an effort to address this issue, we developed a translational control system to regulate gene expression in Chlamydia using a synthetic riboswitch. Here we demon-

\section{Open AcCess}

Citation: Grieshaber NA, Chiarelli TJ, Appa CR, Neiswanger G, Peretti K, Grieshaber SS (2022) Translational gene expression control in Chlamydia trachomatis. PLOS ONE 17(1): e0257259. https:// doi.org/10.1371/journal.pone.0257259

Editor: Jorn Coers, Duke University School of Medicine, UNITED STATES

Received: August 25, 2021

Accepted: January 12, 2022

Published: January 27, 2022

Peer Review History: PLOS recognizes the benefits of transparency in the peer review process; therefore, we enable the publication of all of the content of peer review and author responses alongside final, published articles. The editorial history of this article is available here: https://doi.org/10.1371/journal.pone.0257259

Copyright: ๑ 2022 Grieshaber et al. This is an open access article distributed under the terms of the Creative Commons Attribution License, which permits unrestricted use, distribution, and reproduction in any medium, provided the original author and source are credited.

Data Availability Statement: RNA-seq data is available from the NCBl's Sequence Read Archive (SRA) submission number PRJNA756251. All other relevant data are within the paper and its Supporting Information files. strate that translational control via a riboswitch can be used in combination with a wide range of promoters in $C$. trachomatis. The synthetic riboswitch $E$, inducible with theophylline, was used to replace the ribosome binding site of the synthetic promoter T5-lac, the native chlamydial promoter of the pgp4 plasmid gene and an anhydrotetracycline responsive promoter. In all cases the riboswitch inhibited translation, and high levels of protein expression was induced with theophylline. Combining the Tet transcriptional inducible promoter with the translational control of the riboswitch resulted in strong repression and allowed for the cloning and expression of the potent chlamydial regulatory protein, HctB. The ability to control the timing and strength of gene expression independently from promoter specificity is a new and important tool for studying chlamydial regulatory and virulence genes.

\section{Introduction}

The bacterial species Chlamydia trachomatis (Ctr), are a group of human pathogens composed of over 15 distinct serovars causing trachoma, the leading cause of preventable blindness, and sexually acquired infections of the urogenital tract. According to the CDC, $\mathrm{Ctr}$ is the most frequently reported sexually transmitted infection in the United States, costing the American healthcare system nearly $\$ 2.4$ billion annually $[1,2]$. These infections are widespread among all age groups and ethnic demographics, infecting $~ 3 \%$ of the human population worldwide [3]. In women, untreated genital infections can result in devastating consequences such as pelvic inflammatory disease, ectopic pregnancy, and infertility $[4,5]$. Every year, there are over 4 million new cases of Ctr in the United States [6,7] and an estimated 152 million cases worldwide [8]. Understanding the genetic factors that mediate infection and disease has historically been hindered by the lack of good genetic tools. This has changed dramatically in the last few years with advances in chlamydial transformation. The ability to introduce genetically 
Funding: This work was supported by National Institutes of Health Granst R01Al130072, R21Al135691 and R21Al113617 (NG, SG). The funders had no role in study design, data collection and analysis, decision to publish, or preparation of the manuscript.

Competing interests: The authors have declared that no competing interests exist. manipulatable plasmids into $C t r$ has created multiple opportunities to bring genetic manipulation techniques to the field [9-11]. The ability to alter the expression levels and timing of proteins involved in chlamydial pathogenesis is an important tool in teasing apart the mechanisms that control chlamydial infections.

Here we demonstrate the use of an inducible translational control system using a synthetic riboswitch in Ctr. Riboswitches are naturally occurring mRNA elements that regulate gene expression in all domains of life [12]. In bacteria, riboswitches generally function to interfere with translation of the mRNA. Riboswitches contain an aptamer sequence that binds a cognate ligand causing the mRNA to adopt an alternative secondary-structure conformation. In bacteria, the changes in mRNA secondary structure can control the availability of the ribosome binding site on the mRNA. A collection of synthetic riboswitches was recently developed through screening and rational design [13]. These riboswitches respond to theophylline, a caffeine analog, and function through a translation initiation mechanism. We successfully adapted one of these theophylline inducible riboswitches, termed $\mathrm{E}$ riboswitch, to control gene expression in Ctr. Additionally, we demonstrated that translational control can be used in conjunction with constitutive promoters, inducible promoters and native chlamydial promoters, demonstrating the versatility of translational inducible control of gene expression in a variety of use cases.

\section{Material and methods}

\section{Cell culture}

Cell lines were obtained from the American Type Culture Collection. Cos-7 cells (CRL-1651) were grown in RPMI-1640, supplemented with $10 \% \mathrm{FBS}$ and $10 \mu \mathrm{g} / \mathrm{mL}$ gentamicin (Cellgro). Chlamydia trachomatis serovar L2 (LGV Bu434) was grown in Cos-7 cells. Elementary Bodies (EBs) were purified by density gradient (DG) centrifugation essentially as described [14] following $43-45 \mathrm{~h}$ of infection. EBs were stored at $-80^{\circ} \mathrm{C}$ in Sucrose Phosphate Glutamate (SPG) buffer (10 mM sodium phosphate [8mM K2HPO4, 2mM KH2PO4], $220 \mathrm{mM}$ sucrose, 0.50 mM l-glutamic acid, $\mathrm{pH}$ 7.4) until use.

\section{Vector construction}

All Ctr expression constructs used p2TK2-SW2 [15] as the backbone and cloning was performed using the In-fusion HD EcoDry Cloning kit (FisherScientific). Primers and geneblocks (gBlocks) were ordered from Integrated DNA Technologies (IDT) and are noted in S1 Table. All constructs are penicillin (bla) resistant except p2TK2-SW2-euoprom-ngLVA which is spectinomycin (aadA) resistant.

p2TK2-SW2-T5-E-clover-3xflag. An E-clover-3xFlag fragment was ordered as a gBlock and inserted between the T5 promoter and IncD terminator of p2TK2-SW2 to generate p2TK2-SW2-E-clover-3xFlag. The backbone was generated using primers 5' E-clover-Flag bb and 3' E-clover-Flag bb.

p2TK2-SW2-E-hctB-3xFlag. The hctB ORF was amplified from Ctr L2(434) using the primers indicated in S1 Table. The fragment was used to replace Clover in p2TK2-SW2- E-clover-3xFlag. The primers used to generate the backbone are described in S1 Table.

p2TK2-SW2-Tet-J-E-clover-3xflag. A gBlock encoding the Tet repressor, Tet promoter and the riboJ ribozyme insulator (S1 Table) was inserted upstream of the E riboswitch of p2TK2-SW2-E-clover-3xFlag, replacing the T5 promoter.

p2TK2-SW2-Tet-J-E-hctB-3xFlag. The hctB ORF was amplified from Ctr L2(434) using the primers 5' Tet-J-HctBi and 3' Tet-J-HctBi. The fragment was used to replace Clover in p2TK2-SW2 -Tet-J-E-clover-3xFlag. 
p2TK2-SW2-nprom-E-pgp4-3xFlag. An E-pgp4-3xFlag fragment was ordered as a gBlock and inserted between the pgp4 native promoter and the IncD terminator of p2TK2-SW2. The backbone was generated using the primers indicated in S1 Table.

p2TK2-SW2-T5-E-ngLVA-3xFlag and p2TK2-SW2-Tet-J-E-ngLVA-3xFlag. A neongreenLVA (ngLVA) fragment was ordered as a gBlock from IDT and inserted to replace Clover of both p2TK2-SW2-E-clover-3xFlag and p2TK2-SW2-Tet-J-E-clover-3xflag. The primers indicated in $\mathrm{S} 1 \mathrm{Table}$ were used for both plasmids to generate the back bone.

p2TK2-SW2-euoprom-ngLVA. The primers 5' ngLVAi and 3' ngLVAi (S1 Table) were used to amplify the ngLVA fragment from E-ngLVA-3xFlag and inserted to replace Clover of p2TK2-SW2-euoprom-Clover (aadA) described by Chiarelli et al. [16]. The primers indicated in S1 Table were used to generate the back bone.

\section{Chlamydial transformation and isolation}

Transformation of Ctr L2 was performed essentially as previously described [17]. Briefly, $1 \times 10^{8} \mathrm{EBs}+>2 \mu \mathrm{g}$ DNA/well were used to infect a 6 well plate. Transformants were selected over successive passages with $1 \mathrm{U} / \mathrm{ml}$ penicillin $\mathrm{G}$ or $500 \mu \mathrm{g} / \mathrm{ml}$ spectinomycin as appropriate for each plasmid. The new strain was clonally isolated via successive rounds of inclusion isolation (MOI, $<1$ ) using a micromanipulator. Clonality of each strain was confirmed by isolating the plasmid, transforming into E. coli and sequencing six transformants.

\section{Fluorescence staining}

Cos7 cells on coverslips were infected with the indicated strains. Protein expression regulated by the E-riboswitch only was induced at $16 \mathrm{hpi}$ with $0.5 \mathrm{mM}$ theophylline (dissolved in RPMI media) (Acros Organics, Thermo Scientific ${ }^{\mathrm{TM}}$ ). Protein expression regulated by both the Tet promoter and the E-riboswitch were induced at $16 \mathrm{hpi}$ with $0.5 \mathrm{mM}$ theophylline and $30 \mathrm{ng} / \mathrm{ml}$ anhydroTetracycline (Acros Organics, Thermo Scientific ${ }^{\mathrm{\tau M}}$ ). Theophylline (theo) was dissolved in RPMI media to a concentration of $50 \mathrm{mM}$ and diluted 1:100 to induce protein expression. AnhydroTetracycline (aTc) was dissolved in DMSO to $10 \mathrm{mg} / \mathrm{ml}$ and diluted to $30 \mathrm{ng} / \mathrm{ml}$ in RPMI for protein expression. DMSO diluted 1:333,333 in RPMI served as vehicle control when appropriate. Samples were fixed with $4 \%$ buffered paraformaldehyde at $24 \mathrm{hpi}$ and stained with Monoclonal anti-Flag M2 antibody (1:500, Sigma, Thermo Scientific $\left.{ }^{\mathrm{TM}}\right)$ and alexa 488 anti-mouse secondary antibody to visualize expressing Chlamydia. DAPI was used to visualize DNA. Coverslips were mounted on a microscope slide with a MOWIOL $\mathbb{R}$ mounting solution (100 mg/mL MOWIOLß 4-88, 25\% glycerol, $0.1 \mathrm{M}$ Tris $\mathrm{pH} 8.5$ ).

Fluorescence images were acquired using a Nikon spinning disk confocal system with a $60 \mathrm{x}$ oil-immersion objective, equipped with an Andor Ixon EMCCD camera, under the control of the Nikon elements software. Images were processed using the image analysis software ImageJ (http://rsb.info.nih.gov/ij/). Representative confocal micrographs displayed in the figures are maximal intensity projections of the 3D data sets, unless otherwise noted.

\section{Live cell imaging}

Infected monolayers of Cos7 cells grown in a glass bottom 24 well plate were induced at 16 hpi with either $0.5 \mathrm{mM}$ theophylline only or the indicated concentrations of theophylline and anhydroTetracycline. Plates were imaged immediately upon induction.

Live cell imaging was achieved using an automated Nikon epifluorescent microscope equipped with an Okolab (http://www.oko-lab.com/live-cell-imaging) temperature controlled stage and an Andor Zyla sCMOS camera (http://www.andor.com). Images were taken every fifteen minutes for a further 36 hours. Multiple fields of view of multiple wells were imaged. 
The fluorescence intensity of each inclusion over time was tracked using the ImageJ plugin Trakmate [18]. and the results were averaged and plotted using python and matplotlib [19].

\section{Replating assay}

Ctr were isolated by scraping the infected monolayer into media and pelleting at $17200 \mathrm{rcfs}$. The EB pellets were resuspended in RPMI via sonication and seeded onto fresh monolayers in a 96-well microplate in a 2-fold dilution series. Infected plates were incubated for 24 hours prior to fixation with methanol and stained with DAPI and Ctr MOMP Polyclonal Antibody, FITC (Fishersci). The DAPI stain was used for automated microscope focus and visualization of host-cell nuclei and the anti-Ctr antibody for visualization of EBs and inclusion counts. Inclusions were imaged using a Nikon Eclipse TE300 inverted microscope utilizing a scopeLED lamp at 470nm and 390nm, and BrightLine band pass emissions filters at 514/30nm and 434/17nm. Image acquisition was performed using an Andor Zyla sCMOS in conjunction with $\mu$ Manager software. Images were analyzed using ImageJ software and custom scripts. Statistical comparisons between treatments were performed using an ANOVA test followed by Tukey's Honest Significant Difference test.

\section{Western analysis}

Infected monolayers were lysed in reducing lane marker sample buffer and protein lysates were separated on $12 \%$ SDS-PAGE gels and transferred to a Nitrocellulose Membrane for western analysis of the Flag-tagged protein or $\beta$-tubulin I as a loading control. The membrane was blocked with PBS $+0.1 \%$ Tween 20 (PBS-T) and 5\% nonfat milk prior to incubating in either monoclonal anti-Flag M2 antibody (1:40,000, Sigma, Thermo Scientific $\left.{ }^{\mathrm{m}}\right)$ or anti-beta I Tubulin, Clone: SAP.4G5 (1:20,000, Novus Biologicals ${ }^{\mathrm{Tw}}$, Fishersci) overnight at $4^{\circ} \mathrm{C}$ followed by Goat-anti Mouse IgG-HRP secondary antibody (Invitrogen ${ }^{\mathrm{mm}}$ ) at room temperature for 2 hours. The membrane was developed with the Supersignal West Dura luminol and peroxide solution (Thermo Scientific $c^{\mathrm{Tw}}$ ) and imaged using an Amersham Imager 600.

\section{Glycogen staining}

Monolayers were infected with the indicated strains and induced with $0.5 \mathrm{mM}$ theophylline at the time of infection. At $36 \mathrm{hpi}$, the media was removed and the samples were stained with 1 $\mathrm{ml}$ of a 1:50 dilution of 5\% iodine stain (5\% potassium iodide and 5\% iodine in $50 \%$ ethanol) in PBS for $10 \mathrm{~min}$. Samples were then stained in 1:50 Lugol's iodine solution in PBS (10\% potassium iodide and 5\% iodine in $\mathrm{ddH} 2 \mathrm{O}$ ) and imaged directly. Images were acquired using a Nikon microscope using phase brightfield illumination and an Andor Zyla sCMOS camera.

\section{RNA-Seq}

Total RNA was isolated from cells infected with L2-Tet-J-E-hctB-flag. Expression of HctB was induced with $0.5 \mathrm{mM}$ theophylline and $30 \mathrm{ng} / \mathrm{ml}$ aTc at $15 \mathrm{hpi}$ and the Ctr isolated at $24 \mathrm{hpi}$ on ice. Briefly, the infected monolayer was scraped into ice cold PBS, lysed using a Dounce homogenizer and the $\mathrm{Ctr}$ isolated over a 30\% MD-76R pad. Total RNA was isolated using TRIzol reagent (Life Technologies) following the protocol provided and genomic DNA removed (TURBO DNA-free Kit, Invitrogen). The enriched RNA samples were quantified and the libraries built and barcoded by the IBEST Genomics Resources Core at the University of Idaho. The libraries were sequenced by University of Oregon sequencing core using the Illumina NovaSeq platform. RNA-seq reads were aligned to the published C. trachomatis L2 Bu 434 genome using the bowtie2 aligner software [20]. Reads were quantified using HTseq [21] 
Statistical analysis and normalization of read counts was accomplished using DESeq2 in $\mathrm{R}$ [22]. Log2fold change and statistics were also calculated using DESeq2. Heatmaps and hierarchical clustering were generated and visualized using python with pandas and the seaborn visualization package [23]. Aligned reads are accessible from the NCBI's Sequence Read Archive (SRA) submission number PRJNA756251.

\section{Results}

\section{Translational control of gene expression from a synthetic constitutive promoter}

Controlling the timing and level of gene expression is an important tool for uncovering the function of genes that are involved in chlamydial pathogenesis. We developed an inducible expression system for use in $C$. trachomatis using a synthetic riboswitch that binds the small molecule theophylline $[23,24]$. We used the synthetic riboswitch $\mathrm{E}$ behind a T5-lac promoter (T5) to drive expression of the GFP variant, Clover [25, 26] (Fig 1A). The T5-lac promoter is a hybrid promoter made from the phage $\mathrm{T} 5$ early promoter and the lac-operon [27]. The $\mathrm{E}$ riboswitch when not bound to theophylline folds to block the initiation of translation [28]. However, when the riboswitch binds theophylline the ribosome binding site is no longer obscured by the RNA secondary structure allowing for efficient translation. A T5-E-clover$3 x$ Flag fragment was cloned into the chlamydial plasmid p2TK2-SW2 $[15,29]$ to make the p2TK2-SW2-T5-E-clover-3xflag plasmid (Fig 1A) and transformed into Ctr L2 resulting in the strain L2-E-clover-flag. Cos-7 cells were infected with these transformants and Clover expression was evaluated by western blotting. Cells were infected and treated with either theophylline or vehicle at 16 hours post infection (hpi) and cell lysates were analyzed for protein production at $30 \mathrm{hpi}$. Clover expression was tightly regulated and was only detectable in the theophylline treated sample (Fig1B). In addition to western blotting we also evaluated the expression of Clover using confocal microscopy. Cos-7 cells grown on coverslips were infected with the L2-Eclover-flag strain and Clover expression was induced with theophylline at $16 \mathrm{hpi}$. The coverslips were fixed at $30 \mathrm{hpi}$ and imaged for Clover expression using confocal microscopy. Again, only inclusions treated with theophylline had fluorescent Ctr (Fig 1C).

To determine the effects of theophylline and gene expression induction on chlamydial growth dynamics the production of infectious EBs using a reinfection inclusion forming unit assay was performed. Cos-7 cells were infected with L2-E-clover-flag and induced with theophylline at 16 hpi. EBs were harvested at $30 \mathrm{hpi}$ and $48 \mathrm{hpi}$. Clover induction with theophylline had no significant effect on EB production at $30 \mathrm{hpi} \mathrm{(S1} \mathrm{Fig)} \mathrm{or} 48 \mathrm{hpi}$ (Fig 1D). The control of expression of ectopic proteins to assess their function in pathogenesis needs to be highly customizable as too little or too high concentrations may mask the phenotype of interest. Therefore we assessed the dose responsiveness of the E riboswitch to its ligand theophylline. Gene expression was measured using live-cell time-lapse microscopy and particle tracking to quantify the fluorescent expression of individual inclusions over time [19, 30]. This technique allows for the tracking of gene expression in multiple individual inclusions over the entire developmental cycle. Cos-7 cells were plated in a glass bottom 24 well plate and infected with L2-E-clover-flag at an multiplicity of infection (MOI) of $\sim 0.5$. At 16 hpi theophylline at $1 \mathrm{mM}, 0.5 \mathrm{mM}, 0.25 \mathrm{mM}, 0.0125 \mathrm{mM}, 0.00625 \mathrm{mM}$, and $0.00312 \mathrm{mM}$ was added to individual wells to induce Clover expression; images were taken every 15 minutes for 48 hours. Clover expression followed a dose response with almost immediate detection of fluorescence with $1 \mathrm{mM}$ theophylline and a delayed response at the lowest dose, $0.00312 \mathrm{mM}$ (Fig 1E). The response increased through the life of the inclusion; this increase overtime also followed a dose response (Fig 1E). 
A

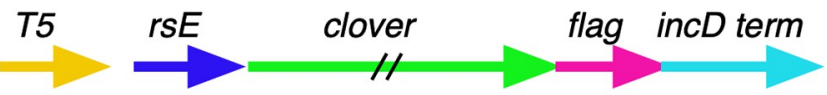

B

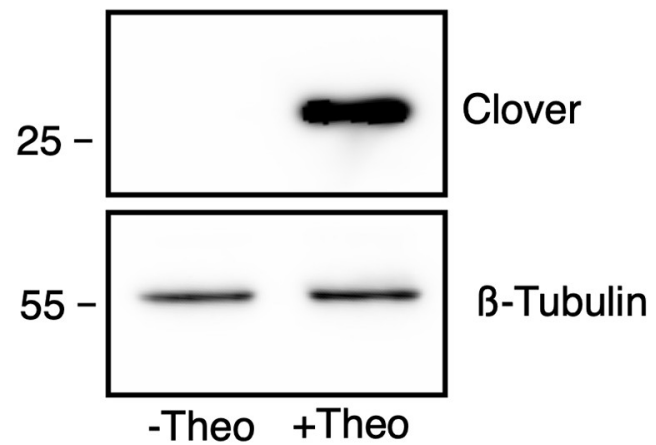

C

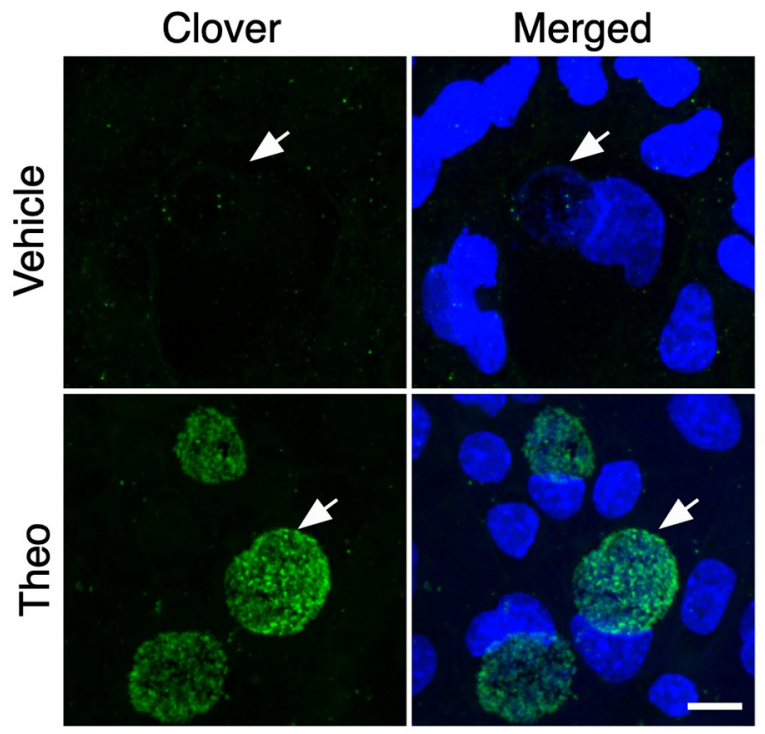

D

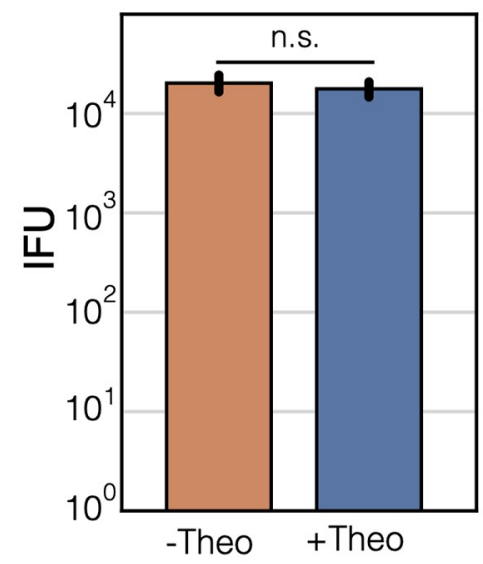

$\mathbf{E}$

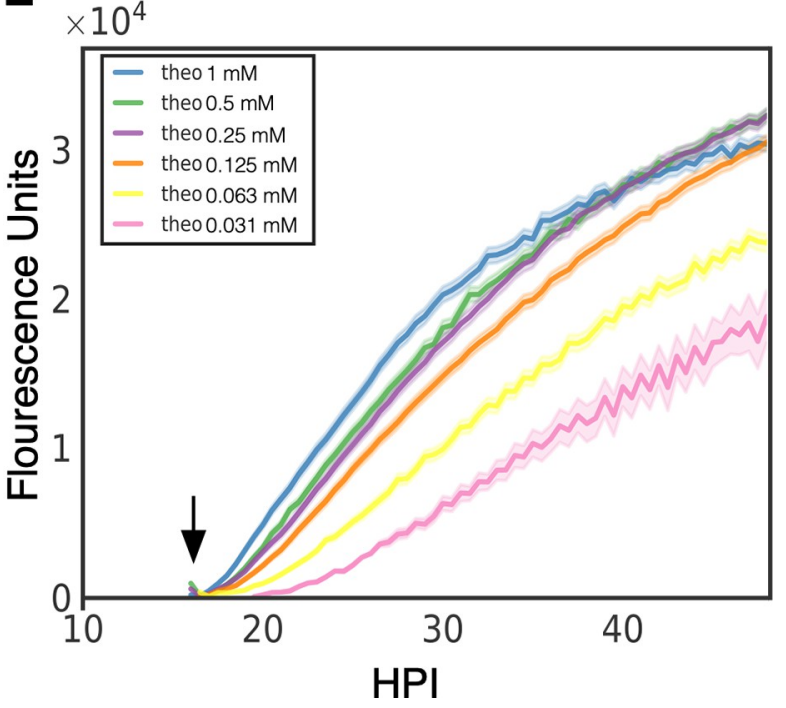

Fig 1. Characterization of p2TK2-SW2-T5-E-clover-flag. A) Schematic of the E-clover expression construct consisting of the T5-lac promoter (T5), riboE riboswitch (rsE) and the ORF for the clover fluorescent protein with an inframe $3 \mathrm{x}$ flag tag. B) Anti-flag western blot of Cos-7 cells infected with L2-T5-Eclover-flag comparing expression of theophylline treated and untreated cultures. Cells were induced or not with $0.5 \mathrm{mM}$ theophylline at 16 hpi and proteins were harvested at $30 \mathrm{hpi}$. A ß-tubulin I western blot served as a sample loading control. C) Confocal micrographs of Cos-7 cells infected with L2-T5-E-cloverflag and induced or not with $0.5 \mathrm{mM}$ theophylline at $16 \mathrm{hpi}$ and fixed and stained with DAPI for microscopy at 30 hpi. DAPI (blue), Clover (green). Arrow indicates the position of the chlamydial inclusion. Size bar $=10 \mu \mathrm{m}$. D) Cos-7 cells were infected with L2-T5-E-clover-flag and the production of infectious progeny was determined at $48 \mathrm{hpi}$ after $0.5 \mathrm{mM}$ theophylline induction or vehicle only. E) Cos-7 cells were infected with L2-T5-E-clover-flag, treated with varying dilutions of theophylline at $16 \mathrm{hpi}(1 \mathrm{mM}, 0.5 \mathrm{mM}, 0.25 \mathrm{mM}, 0.125 \mathrm{mM}, 0.0625 \mathrm{mM}, 0.03125 \mathrm{mM})$ and imaged for 50 hours using live cell imaging. The Clover expression intensities from $>50$ individual inclusions were monitored via automated live-cell fluorescence microscopy and average intensities were plotted. Live cell imaging demonstrated that Clover induction was dose responsive. Cloud represents SEM. Y-axes are denoted in scientific notation. Error bars $=$ SEM. n. s. denotes p-values $>0.05$.

\section{Translational control of gene expression from a native chlamydial} promoter

The use of non endogenous promoters for ectopic expression is an important tool for understanding protein function. However, these systems lack the ability to control gene expression through native gene regulation making it difficult to modulate expression at biologically relevant times or in the correct cell subspecies. This is especially true for $\mathrm{Ctr}$ as it proceeds through a time dependent developmental cycle that includes multiple phenotypic cell types. Therefore, 
the use of translational control was tested in concert with a native chlamydial promoter. We tested the effectiveness of translational control on the pgp4 native plasmid gene. Pgp4 is a regulator of other plasmid genes as well as chromosomal genes [31-33]. Ctr strains with pgp4 knocked out from the native plasmid show marked changes in gene expression and a phenotypic loss of glycogen accumulation [33]. To assess the ability to regulate translation of transcripts from a native promoter, the E riboswitch was cloned upstream of the pgp4 open reading frame (ORF) replacing the predicted ribosome binding site (Fig 2A). The insertion was designed to not disrupt the native promoter region of pgp4. A flag tag was also added in frame to the end of the pgp4 ORF creating the plasmid p2TK2-SW2-nprom-E-pgp4-3xflag (Fig 2A). This plasmid was then transformed into Ctr L2 to create L2-nprom-E-pgp4-flag. To assess expression, Cos-7 cells were infected with the L2-nprom-E-pgp4-flag strain in the presence of $0.5 \mathrm{mM}$ theophylline. Expression was assessed by western blotting and flag tag detection was greatly increased in theophylline treated samples (Fig 2B). The control of pgp4 expression was also assessed by confocal microscopy. Cos-7 cells were plated on coverslips and infected with L2-nprom-E-pgp4-flag and treated with $0.5 \mathrm{mM}$ theophylline at infection. The coverslips were fixed at $30 \mathrm{hpi}$, stained with an anti-flag antibody and DAPI for visualization (Fig 2C). Like for the western blotting experiment, flag epitope detection was dramatically increased in the theophylline induced samples (Fig 2C). The effect of modulating pgp4 expression on EB production was determined using a re-infection assay. Cos-7 cells were infected with L2-nprom-E-pgp4-flag and translation was induced at infection with $0.5 \mathrm{mM}$ theophylline. EBs were harvested at 48 hours and monolayers were re-infected and inclusions quantified. Repression of pgp4 expression resulted in a slight but statistically significant increase in infectious progeny as compared to induced pgp4 expression (Fig 2D). Pgp4 positively regulates the expression of GlgA which is involved in accumulation of glycogen in the inclusion. When pgp4 expression is missing the Ctr inclusion does not accumulate glycogen and is phenotypically similar to the plasmidless L2 strain, L2R [33]. Therefore, we tested the ability of translational regulation to control glycogen accumulation in the inclusion. Cos-7 cells were infected with the L2-nprom-E-pgp4-flag strain and treated or not with $0.5 \mathrm{mM}$ theophylline at the time of infection. Cells were stained for glycogen accumulation at 36 hpi using Lugol's iodine solution as previously described [33]. As expected from the flag detection of expression, glycogen staining was strongly detected in inclusions that were treated with theophylline (Fig 2E). The uninduced inclusions were morphology similar to inclusions formed by the plasmidless strain L2R which lack glycogen accumulation (Fig 2E).

\section{Transcriptional and translational control of gene expression}

The E riboswitch partnered with either the T5 promoter or native pgp4 promoter offered very tight expression control. There was no detectable Clover or Pgp4 by western blotting and no fluorescence from Clover or Flag staining detected using confocal microscopy (Figs 1 and 2). However, we attempted to use the T5-E system to ectopically express the Ctr histone like protein HctB. Clover was replaced on the p2TK2-SW2-T5-E-clover-3xflag plasmid with HctB creating p2TK2-SW2-T5-E-hctB-3xflag. This construct was then transformed into Ctr. Although we successfully isolated transformants, when the plasmids were purified and sequenced, the promoter region of the plasmid was mutated in every case. We reasoned that the HctB protein expression was leaky enough to lead to small amounts of HctB accumulation despite the translation inhibition of the E riboswitch, thereby inhibiting the chlamydial developmental cycle. We therefore sought to create an extremely tightly regulated inducible expression system by combining inducible transcription with inducible translation. For this construct we added the Tet repressor and replaced the $\mathrm{T} 5$ promoter with a Tet promoter containing Tet operator sites 
A

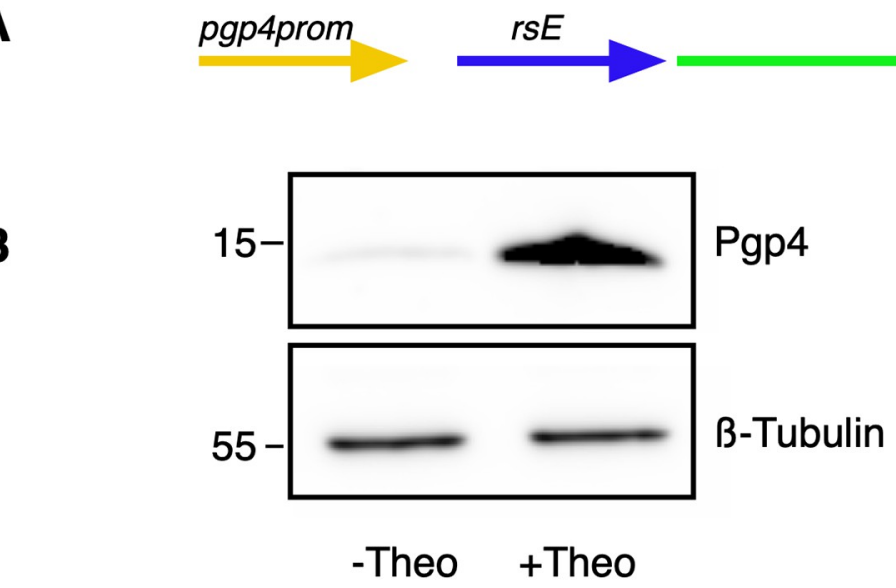

B

C

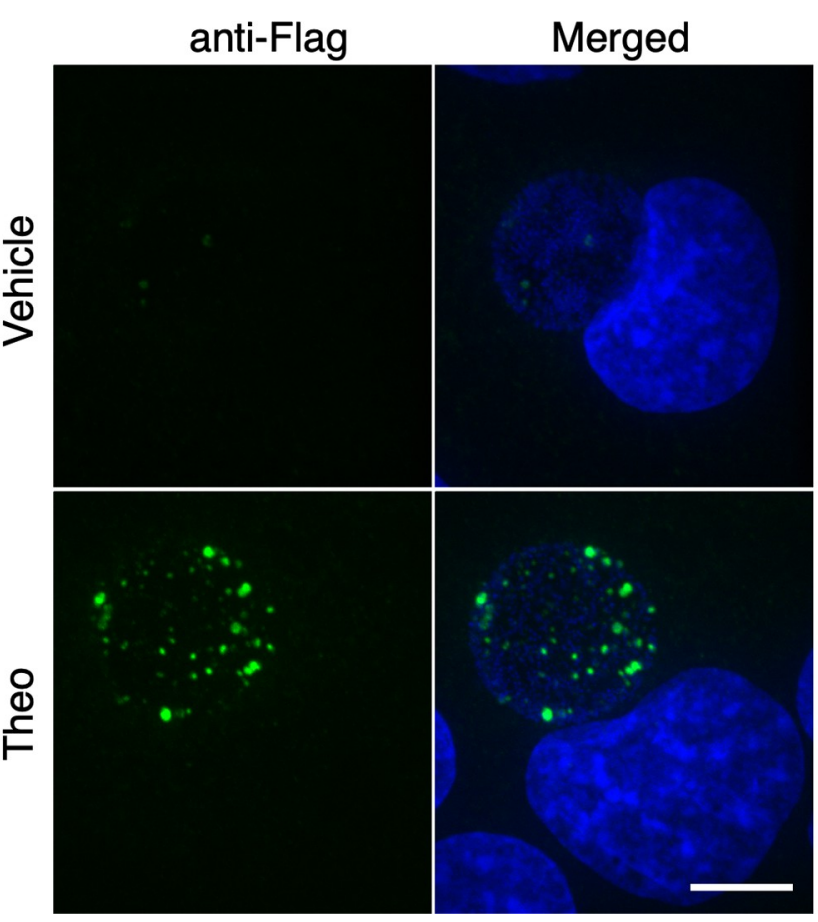

pgp4

ag
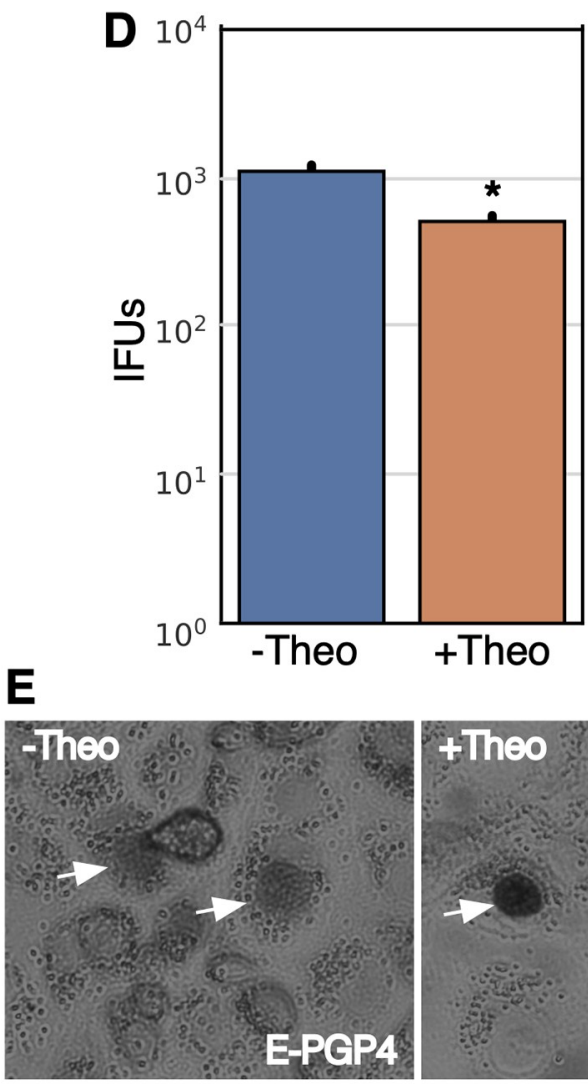

tTheo
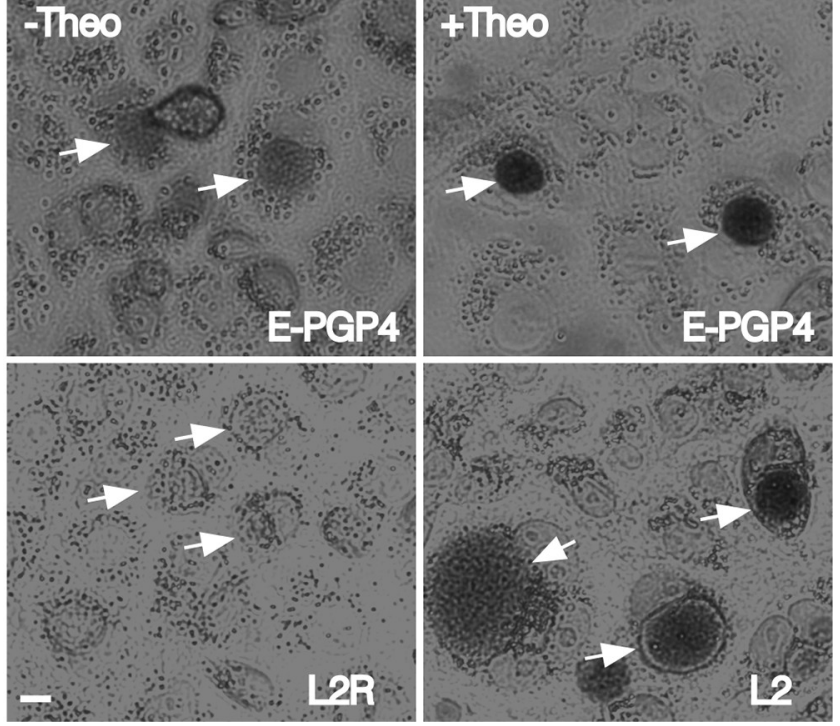

(he nem native pgp4 promoter, the riboE riboswitch ( $\mathrm{rsE}$ ), and the pgp4 ORF with an inframe 3x flag tag. B) Anti-flag western blot of Cos-7 cells infected with L2-nprom-E-pgp4-flag comparing expression of theophylline treated and untreated cultures. Cells were induced or not with $0.5 \mathrm{mM}$ theophylline at $16 \mathrm{hpi}$ and proteins were harvested at $30 \mathrm{hpi}$ A B-tubulin I western blot served as a loading control. C) Confocal micrographs of Cos-7 cells infected with L2-nprom-E-pgp4-flag, induced or not with 0.5 $\mathrm{mM}$ theophylline at $16 \mathrm{hpi}$ and fixed and stained with DAPI to detect DNA. The flag tag was detected using a primary antibody to the tag and an alexa 488 antimouse secondary antibody (green). Size bar $=10 \mu \mathrm{m}$. D) Cos-7 cells were infected with L2-nprom-E-pgp4-flag and the production of infectious progeny was determined at 48 hpi after $0.5 \mathrm{mM}$ theophylline induction or vehicle only. E) Iodine staining of glycogen in the inclusion of Cos-7 cells infected with

L2-nprom-E-pgp4-flag after $0.5 \mathrm{mM}$ theophylline induction at $16 \mathrm{hpi}$ or vehicle only. Arrows indicate the location of the chlamydial inclusions. Asterisk denotes $\mathrm{p}$-value $<0.05$. Error bars $=$ SEM.

https://doi.org/10.1371/journal.pone.0257259.g002

in the p2TK2-SW2-T5-E-clover-3xflag plasmid [34] (Fig 3A). In addition to replacing the T5 promoter with the Tet promoter, a ribozyme insulator was added to the E riboswitch to decouple the promoter from the riboswitch (Fig 3A). The riboJ ribozyme insulator is a self cleaving 75 nucleotide sequence from the satellite RNA of tobacco ringspot virus (sTRSV) followed by a 23 nucleotide hairpin [35]. After transcription, the ribozyme self-cleaved, removing 
A

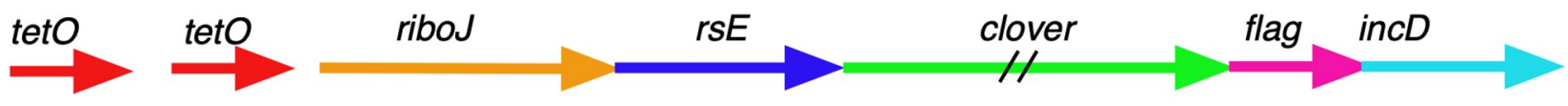

B
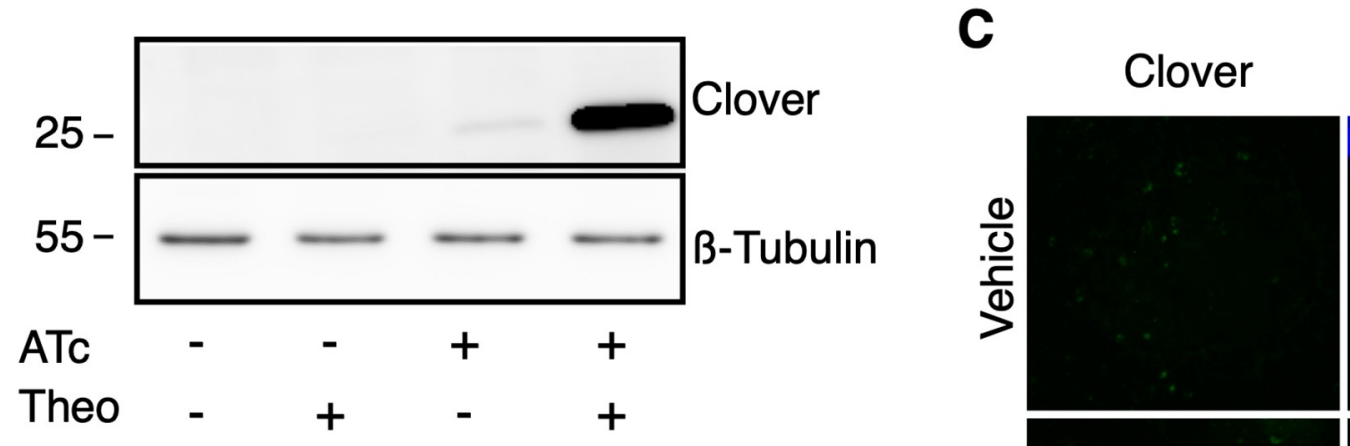

Merged

D
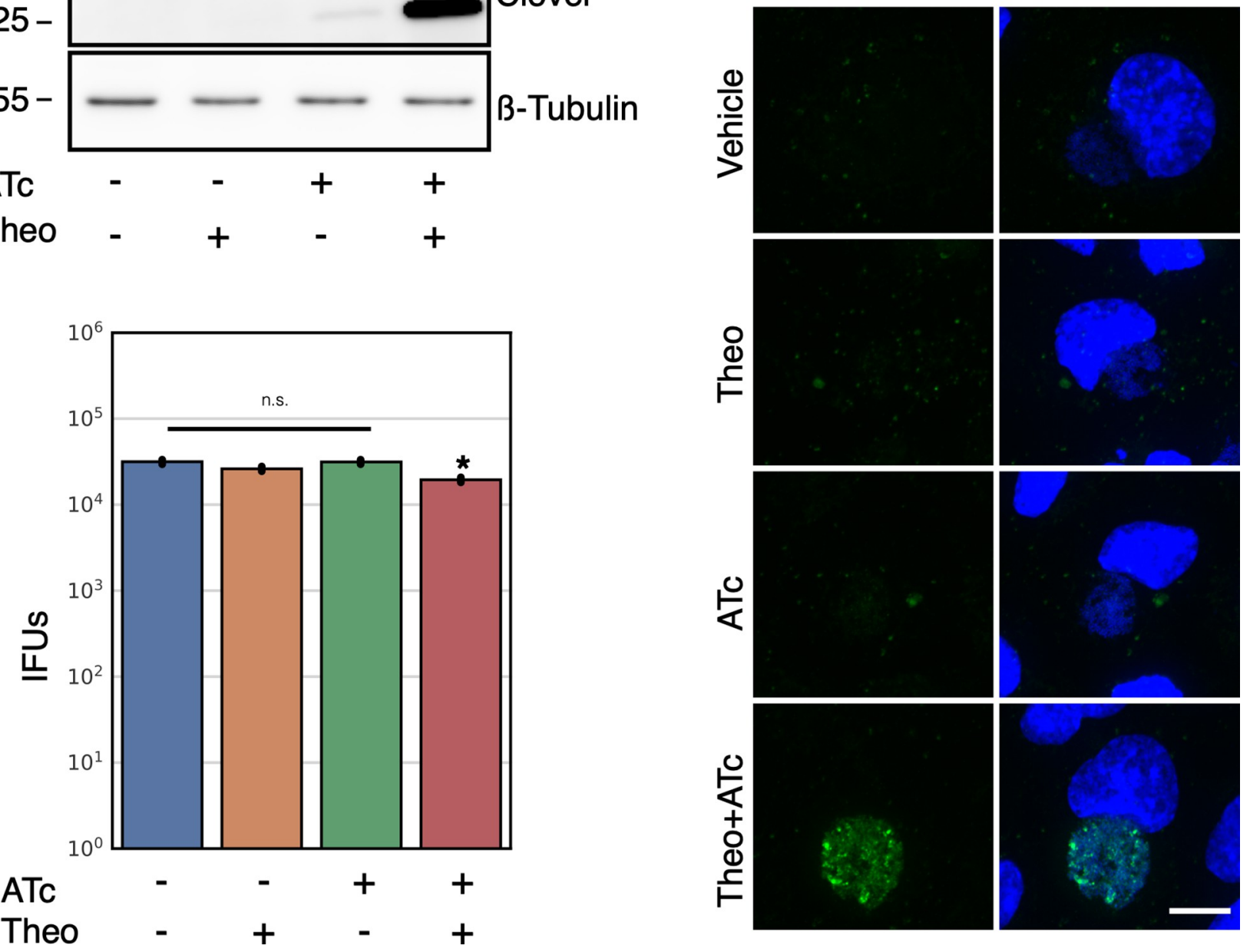

Fig 3. Characterization of p2TK2-SW2-Tetp-riboJ-E-clover-flag. A) Schematic of the Tet-riboJ-E-clover-flag construct consisting of the tet repressor, tet promoter, riboJ insulator, riboE riboswitch (rsE) and the ORF for the clover fluorescent protein containing an inframe $3 \mathrm{x}$ flag tag. B) Anti-flag western blot of Cos-7 cells infected with L2-Tet-J-E-clover-flag comparing expression of theophylline treated and untreated cultures. Cells were induced with $0.5 \mathrm{mM}$ theophylline, $30 \mathrm{ng} / \mathrm{ml} \mathrm{aTc}$, both aTc and theophylline or vehicle only at $16 \mathrm{hpi}$ and proteins were harvested at $30 \mathrm{hpi}$. An anti- $\beta$-tubulin I western blot served as a loading control. C) Confocal micrographs of Cos-7 cells infected with L2-Tet-J-E-clover-flag, induced with $0.5 \mathrm{mM}$ theophylline, 30ng/ml aTc, both aTc and theophylline or vehicle only at $16 \mathrm{hpi}$ and fixed and stained with DAPI (blue) for confocal microscopy at $30 \mathrm{hpi}$. Clover expression (green) was evident in cells treated with aTc and Theophylline. Size bar $=10 \mu \mathrm{m}$. D) Cos-7 cells were infected with L2-Tet-J-E-clover-flag and the production of infectious progeny was determined at $48 \mathrm{hpi}$ after induction with $0.5 \mathrm{mM}$ theophylline, $30 \mathrm{ng} / \mathrm{ml} \mathrm{aTc}$, both aTc and theophylline or vehicle only at 16 hpi. Production of infectious progeny was determined using a reinfection assay. Asterisk denotes $\mathrm{p}$-value $<0.05$. Error bars $=$ SEM.

https://doi.org/10.1371/journal.pone.0257259.g003

upstream sequences, eliminating the promoter-associated RNA leader (S2 Fig). This resulted in transcripts with a small hairpin region just upstream of the E riboswitch that we hypothesized would not affect the aptamer function of the riboswitch. We used this same regulatory scheme to control the expression of both Clover and HctB resulting in plasmids p2TK2-SW2-Tet-J-E-clover-3xflag and p2TK2-SW2-Tet-J-E-hctB-3xflag. For the HctB clone 
we used the AUG start site and the first three codons of the Clover gene followed by the HctB ORF without the AUG. We chose to use the first three codons of Clover as some genes in Ctr have small RNA regulatory sites at the beginning of the gene and wanted to avoid any native regulation [36]. These constructs were transformed into Ctr creating the strains L2-Tet-J-Eclover-flag and L2-Tet-J-E-hctB-flag.

Expression of Clover from p2TK2-SW2-Tet-J-E-clover-3xflag was evaluated by western blotting. Cos-7 cells were infected with the L2-Tet-J-E-clover-flag strain and expression was induced with the addition of either anhydroTetracycline (aTc) $30 \mathrm{ng} / \mathrm{ml}$ or theophylline 0.5 $\mathrm{mM}$ alone, or both combined at $16 \mathrm{hpi}$. At 30 hpi protein from the infected cells was harvested, separated by PAGE and blotted to nitrocellulose for detection. Clover expression was detected using an anti-flag antibody. As expected Clover expression was only detected in samples induced with both aTc and theophylline (Fig 3B). We also evaluated Clover expression using confocal microscopy. Cos-7 cells plated on glass coverslips were infected for 16 hours before induction of expression with aTc $30 \mathrm{ng} / \mathrm{ml}$, theophylline $0.5 \mathrm{mM}$ or both combined. The coverslips were fixed at $30 \mathrm{hpi}$, stained with DAPI and visualized by confocal microscopy. Again, robust Clover expression was only evident when both transcription and translation were induced (Fig 3C).

The effects of induction of this system was evaluated for effects on the chlamydial developmental cycle. The impact of induction on the production of infectious EBs was measured using an inclusion forming reinfection assay (IFU). Cos-7 cells were infected with L2-Tet-J-Eclover-flag and Clover expression was induced with aTc $30 \mathrm{ng} / \mathrm{ml}$, theophylline $0.5 \mathrm{mM}$ or both combined at $16 \mathrm{hpi}$. EBs were harvested at both $30 \mathrm{hpi}$ and $48 \mathrm{hpi}$ to evaluate the production of infectious progeny (Fig 3D and S1 Fig). Each inducer alone had no effect on IFU formation. However, the addition of both inducers had a very small but statistically significant reduction in IFUs suggesting the expression of Clover resulted in a slight impact to the chlamydial developmental cycle (Fig 3D and S1 Fig).

To assess the effects of the induction of transcription or translation order we measured the kinetics of Clover expression using live cell imaging as described earlier. Cos-7 cells plated into 24 well glass bottom plates were infected with L2-Tet-J-E-clover-flag and either treated with aTc $(30 \mathrm{ng} / \mathrm{ml})$ at infection and treated with a decreasing dose of theophylline (2 fold dilutions from $1 \mathrm{mM}$ to $0.0312 \mathrm{mM}$ ) at $16 \mathrm{hpi}$ (Fig $4 \mathrm{~A})$ or theophylline $(0.5 \mathrm{mM})$ at infection followed by a decreasing dose of aTc ( 2 fold dilutions from $60 \mathrm{ng} / \mathrm{ml}$ to $1.25 \mathrm{ng} / \mathrm{ml}$ ) at $16 \mathrm{hpi}$ (Fig 4B). Infected cells were imaged for Clover expression every 30 minutes for 50 hours. Gene expression was quantified using live-cell time-lapse microscopy and particle tracking to quantify the fluorescent expression of individual inclusions over time [19]. Clover expression using transcriptional induction followed by translational induction demonstrated a robust dose response. Expression was detectable almost immediately after theophylline addition and detected at the lowest dose of $0.0312 \mathrm{mM}$ theophylline (Fig 4A). Interestingly, max expression kinetics was observed with $0.5 \mathrm{mM}$ of theophylline while $1 \mathrm{mM}$ resulted in less expression suggesting potential toxicity at high concentrations (Fig 4A). When transcription was induced first (aTc) followed by translational induction at $16 \mathrm{hpi}$, expression was again initiated with little delay and a very strong dose response was observed (Fig 4B). Transcriptional induction with aTc resulted in higher expression as we did not reach a point of toxicity. This resulted in the highest expression being at the highest concentration of aTc $(60 \mathrm{ng} / \mathrm{ml})$ (Fig 4B). No induction was observed at the lowest aTc concentration $(1.25 \mathrm{ng} / \mathrm{ml})$. Notably, transcriptional induction followed by translational induction resulted in slightly higher induction as compared to translational induction followed by transcriptional induction as can be seen by comparing aTc $30 \mathrm{ng} / \mathrm{ml}$ followed by $0.5 \mathrm{mM}$ theophylline at 16 hpi to $0.5 \mathrm{mM}$ theophylline at infection followed by $30 \mathrm{ng} / \mathrm{ml}$ of aTc at $16 \mathrm{hpi}$ (Fig $4 \mathrm{~A}$ and $4 \mathrm{~B}$ ). 
A

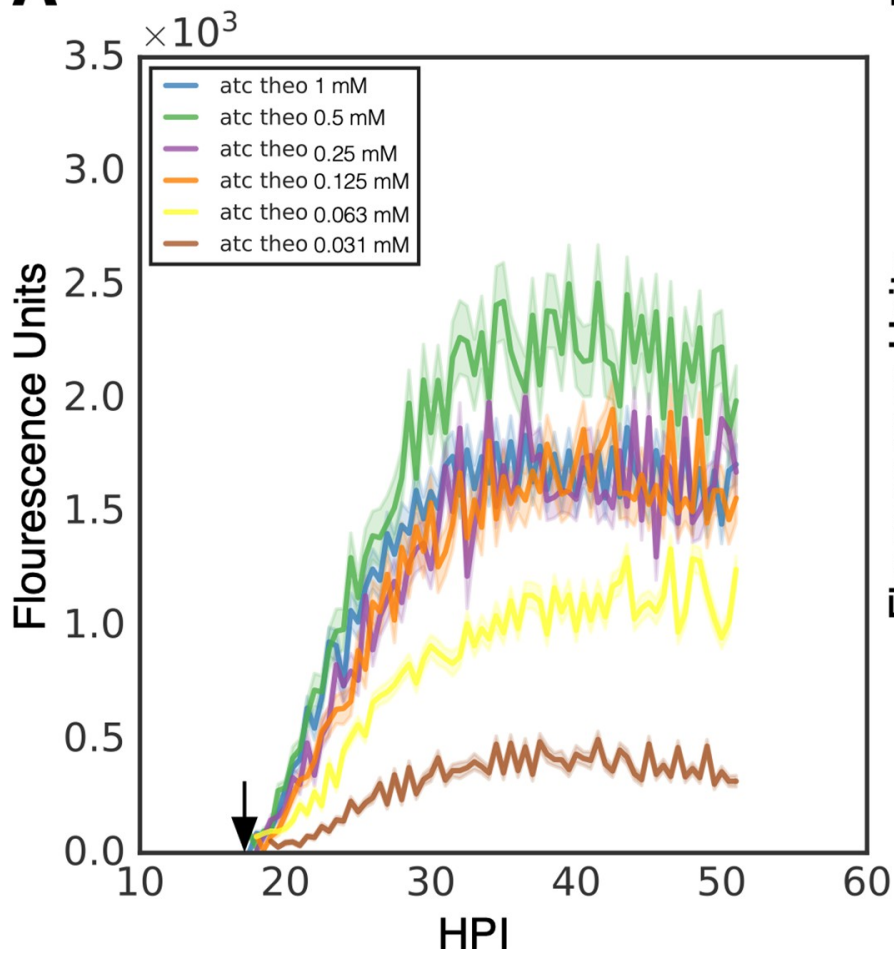

B

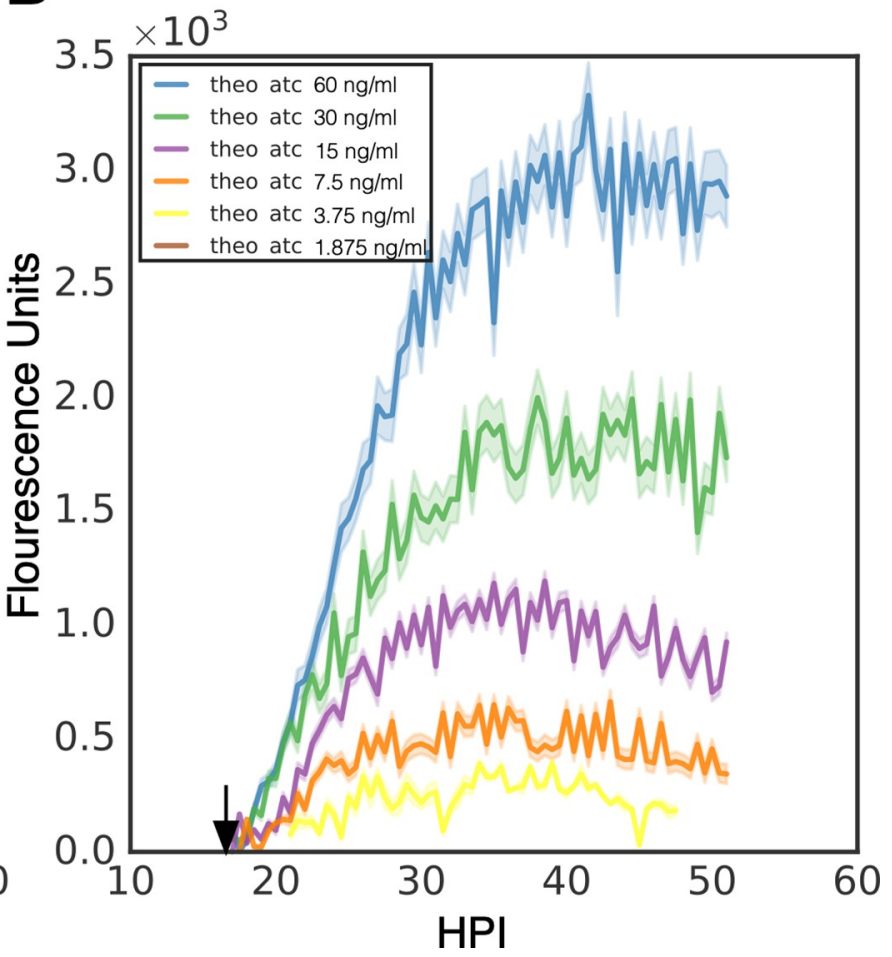

Fig 4. Induction kinetics of p2TK2-SW2-Tet-J-E-clover-flag. A) Cos-7 cells were infected with L2-Tet-J-E-clover-flag, treated with varying dilutions of theophylline at $16 \mathrm{hpi}(1 \mathrm{mM}, 0.5 \mathrm{mM}, 0.25 \mathrm{mM}, 0.125 \mathrm{mM}, 0.0625 \mathrm{mM}, 0.03125 \mathrm{mM})$ and $30 \mathrm{ng} / \mathrm{ml}$ aTc at 0 hpi. The infections were monitored using live cell imaging for 50 hours. B) Cos-7 cells were infected with L2-Tet-riboJ-E-clover-flag, treated with varying dilutions of aTc at $16 \mathrm{hpi}$ ( $60 \mathrm{ng} / \mathrm{ml}, 30 \mathrm{ng} / \mathrm{ml}, 15 \mathrm{ng} /$ $\mathrm{ml}, 7.5 \mathrm{ng} / \mathrm{ml}, 3.75 \mathrm{ng} / \mathrm{m}, 1.875 \mathrm{ng} / \mathrm{m}$ ) and $0.5 \mathrm{mM}$ theophylline at $0 \mathrm{hpi}$. The infections were monitored using live cell imaging for 50 hours. Expression intensities from $>50$ individual inclusions were monitored via automated live-cell fluorescence microscopy and the mean intensities are shown. Cloud represents SEM. Y-axes are denoted in scientific notation.

https://doi.org/10.1371/journal.pone.0257259.g004

To test the effective repression of gene expression of this system p2TK2-SW2-Tet-J-E-hctB3xflag (Fig 5A) was transformed into Ctr producing L2-Tet-J-E-hctB-flag. Unlike the p2TK2-SW2-E-hctB-3xflag construct, p2TK2-SW2-Tet-J-E-hctB-3xflag successfully transformed into $\mathrm{Ctr}$ without accumulating mutations suggesting tighter repression of leaky expression. Cos-7 cells were infected with L2-Tet-J-E-hctB-flag and induced for HctB expression with $30 \mathrm{ng} / \mathrm{ml}$ aTc and $0.5 \mathrm{mM}$ theophylline at 16 hpi. Protein was isolated, separated by PAGE, blotted to nitrocellulose and expression was evaluated using an anti-flag antibody. HctB-flag was detected only when both inducers (aTc and theophylline) were used (Fig 5B). Gene expression was also assessed using confocal microscopy. Cos-7 cells were plated onto glass coverslips and infected with L2-Tet-J-E-hctB-flag. Gene expression was induced with 30 $\mathrm{ng} / \mathrm{ml} \mathrm{aTc}, 0.5 \mathrm{mM}$ theophylline, or both at $16 \mathrm{hpi}$ and the coverslips were fixed and stained with an anti-flag antibody at $30 \mathrm{hpi}$ before mounting for confocal microscopy. Confocal microscopy confirmed HctB induction with both the transcription and translation inducer added (Fig 5C). However, HctB expression was detected at low levels when induced with aTc only suggesting translational repression with this construct was slightly leaky (Fig 5C).

As we could not transform p2TK2-SW2-T5-E-hctB-flag into Chlamydia, we hypothesized that expression of $\mathrm{HctB}$ inhibited the formation of the infectious $\mathrm{EB}$ cell form. To test this, Cos-7 cells were infected with L2-Tet-J-E-hctB-flag and induced with $30 \mathrm{ng} / \mathrm{ml} \mathrm{aTc}, 0.5 \mathrm{mM}$ theophylline, or both at $16 \mathrm{hpi}$ and EBs were harvested at $30 \mathrm{hpi}$ and $48 \mathrm{hpi}$. Induction of both transcription and translation resulted in a greater than $2.5 \log$ reduction in infectious progeny 
A

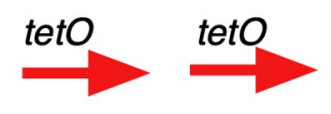

B

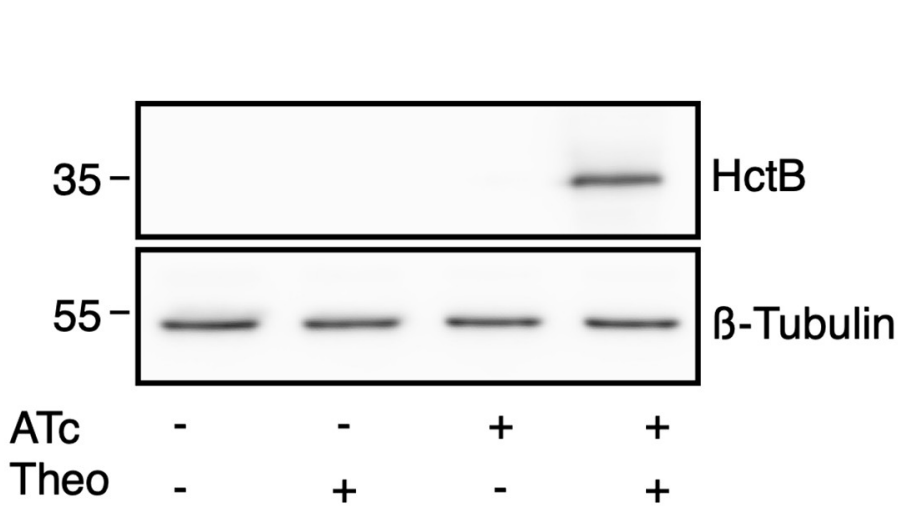

D
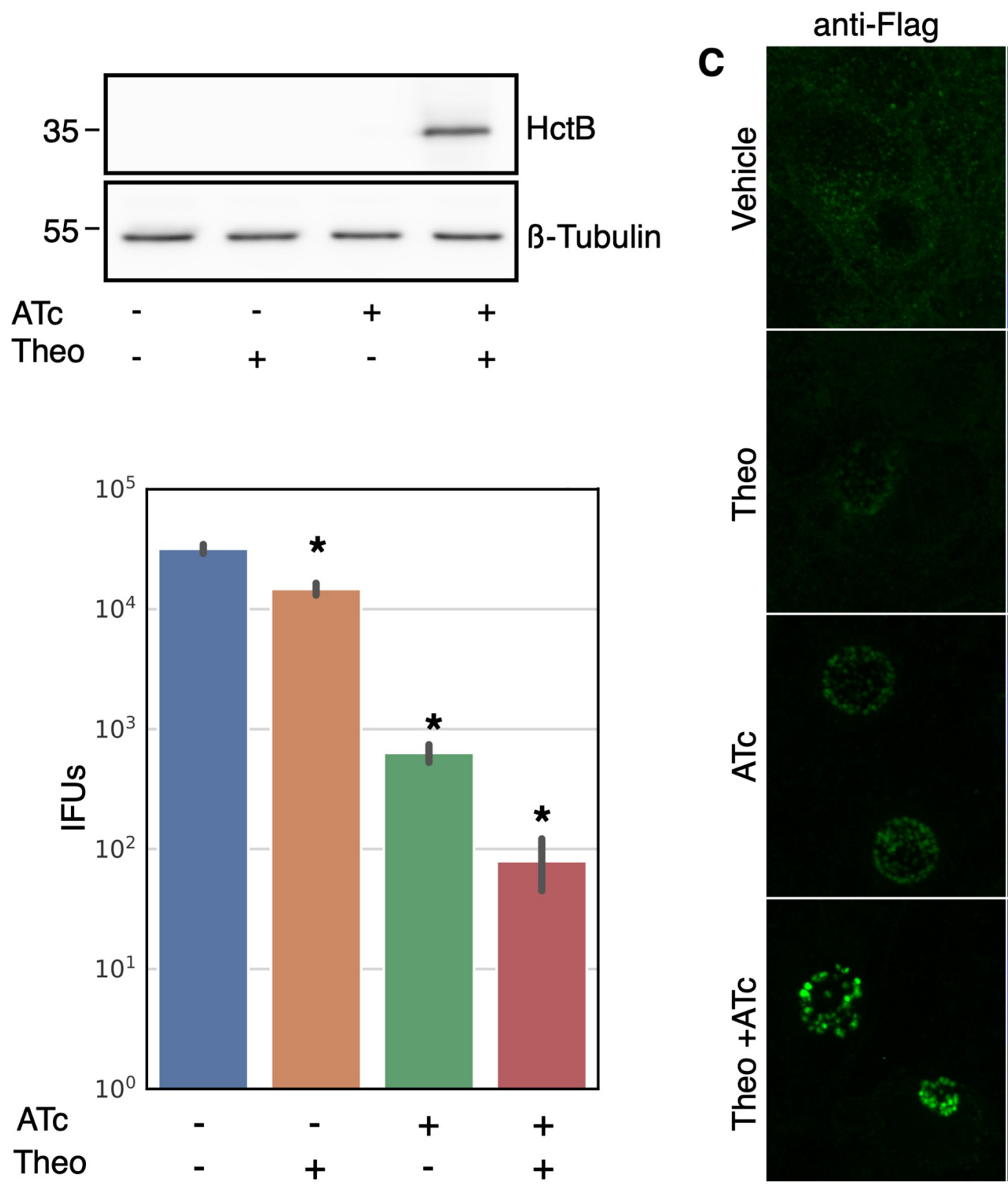

Merged
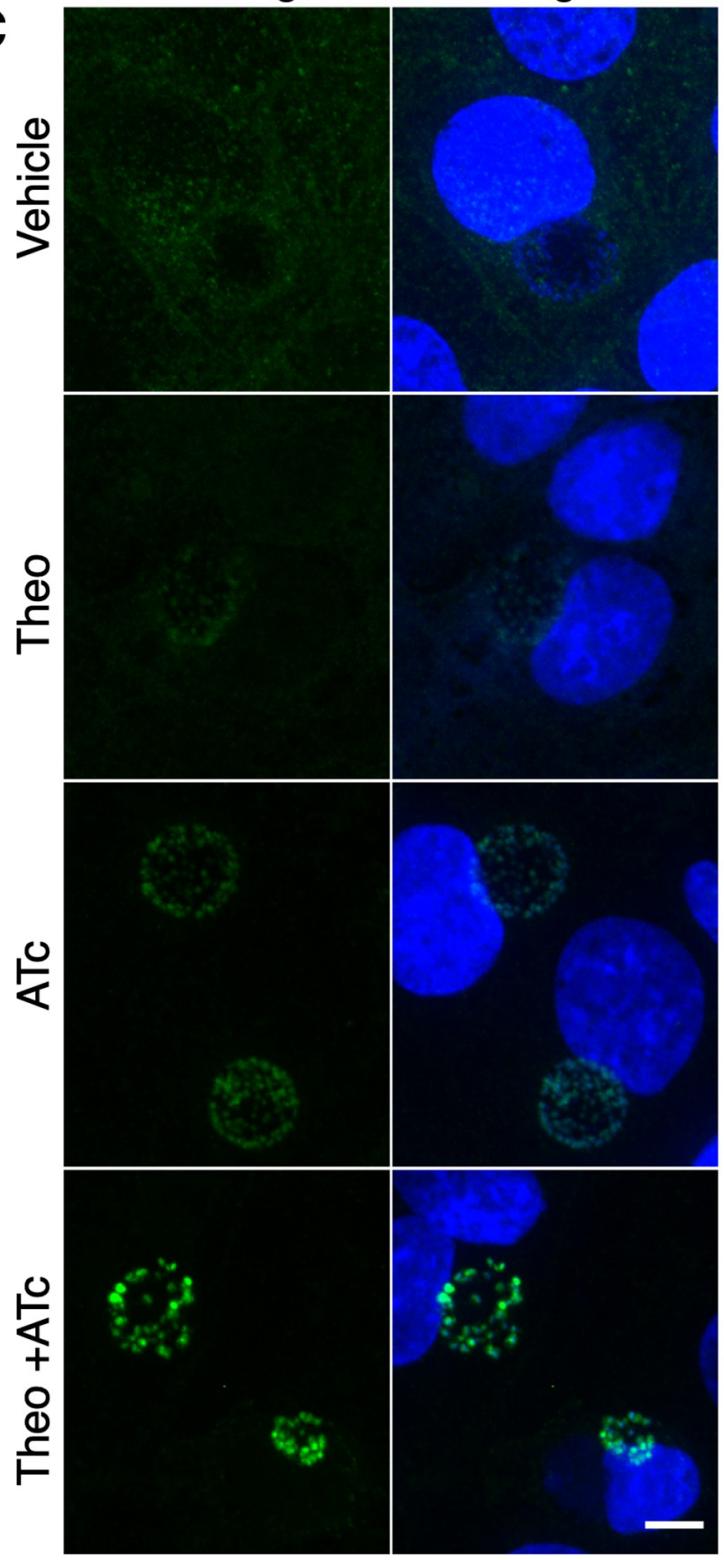

Fig 5. Characterization of p2TK2-SW2-Tet-J-E-hctB-flag. A) Schematic of the Te-riboJ-E-hctB-flag construct. HctB expression is controlled by an aTc inducible promoter, riboJ insulator and the riboE riboswitch. B) Anti-flag western blot of Cos-7 cells infected with L2-Tet-J-E-hctB-flag comparing expression of theophylline treated and untreated cultures. Cells were induced with $0.5 \mathrm{mM}$ theophylline, $30 \mathrm{ng} / \mathrm{ml} \mathrm{aTc}$, both aTc and theophylline or vehicle only at 16 hpi and proteins were harvested at $30 \mathrm{hpi}$. HctB-flag expression was only detected in the samples induced with both aTc and theophylline. C) Confocal micrographs of Cos-7 cells infected with L2-Tet-riboJ-E-hctB-flag, induced with $0.5 \mathrm{mM}$ theophylline, 30ng/ml aTc, both aTc and theophylline or vehicle only at $16 \mathrm{hpi}$ and fixed and stained with DAPI (blue) for confocal microscopy at $30 \mathrm{hpi}$. The flag tag was stained with a primary antibody to the flag and an alexa 488 anti-mouse secondary antibody (green). Size bar $=10 \mu \mathrm{m}$. D) Production of infectious progeny was determined using a reinfection assay. Cos-7 cells were infected with L2-Tet-J-E-hctB-flag and the production of infectious progeny was determined at $48 \mathrm{hpi}$ after induction with $0.5 \mathrm{mM}$ theophylline, $30 \mathrm{ng} / \mathrm{ml}$ aTc, both aTc and theophylline or vehicle only at $16 \mathrm{hpi}$. Asterisks denote p-values $<0.05$. Error bars $=\mathrm{SEM}$.

https://doi.org/10.1371/journal.pone.0257259.g005 
at both $30 \mathrm{hpi}$ and $48 \mathrm{hpi}$ (Fig 5D, S1 Fig). Confocal microscopy indicated that transcription induction with aTc only resulted in low but detectable HctB production (Fig $5 \mathrm{C}$ ) and this leaky expression was also evident when assaying for infectious progeny. Induction of transcription only resulted in about a log reduction in infectious progeny at both $30 \mathrm{hpi}$ and $48 \mathrm{hpi}$ (Fig 5D, S1 Fig). Translation induction only resulted in a slight but statistically significant decrease in EB production as compared to no induction control (Fig 5D, S1 Fig). Together these data suggest that the combination of transcriptional repression and translational inducible regulation was significantly tight enabling Ctr to be successfully transformed with the construct and that induction was sufficiently high to induce the inhibition of the production of infectious progeny.

\section{Expression from T5 and Tet-J-E is cell type specific}

Chlamydial infection of vertebrate cells consists of a multiple cell type developmental cycle. For $\mathrm{Ctr}$ L2 the elementary body (EB) cell type mediates cell entry and differentiates into the reticulate body $(\mathrm{RB})$ cell type over an $\sim 10$ hour period before initiating cell division. The RB cell type undergoes growth and division leading to an expansion of RB numbers. The RB cell type also matures during this process eventually producing an intermediate body (IB) cell type that matures back into the EB cell form over $\sim 8$ hours [19]. Our studies have shown that different promoters are active in these distinct cell populations [19]. Confocal microscopy of Clover expression and flag staining for both the T5-E-clover-flag and Tet-J-E-clover-flag constructs appeared non uniform in the inclusion suggesting expression in only a subset of cells. To determine the cells in which these promoters were active we replaced Clover in both of these constructs with the GFP variant Neongreen and added an inframe LVA degradation tag to produce the plasmids p2TK2-SW2-T5-E-ngLVA-3xFlag and p2TK2-SW2-Tet-J-E-ngLVA$3 x$ Flag. Neongreen-LVA (ngLVA) protein had a halflife of $\sim 30$ minutes in Ctr (S3 Fig). The plasmids were transformed into Ctr and the expression pattern of these constructs was compared to that of p2TK2-SW2-euoprom-ngLVA. p2TK2-SW2-euoprom-ngLVA, like p2TK2-SW2-euoprom-Clover [19] uses the euo promoter to drive expression specifically in the RB cell type. Cos-7 cells infected with L2 T5-E-ngLVA, L2 Tet-J-E-ngLVA, or L2 euoprom-ngLVA were fixed for confocal microscopy at $30 \mathrm{hpi}$. Expression of ngLVA for all three promoters was very similar showing expression in a subset of large cells suggestive of RBs (Fig 6A). Quantification of the number of cells per inclusion that expressed ngLVA from each promoter showed that all three promoters expressed ngLVA in similar numbers of cells (Fig 6B). This suggests that both of the synthetic sigma70 optimized promoters (T5 and Tet) when used in Ctr expressed primarily in the RB cell type and not in the IB.

\section{Conclusions}

Ectopic gene expression is an important tool for uncovering the function of potential virulence associated genes in pathogenic bacteria. We have adapted the E riboswitch, a theophylline binding aptamer, to regulate gene translation in Ctr. Riboswitches have been used in many organisms to regulate gene expression [16, 24, 25, 37-39]. In bacteria, riboswitches are constructed of aptamers that fold to block ribosome assembly at the translational start site in the absence of their cognate ligand. This translational control can be combined with strong synthetic promoters, native promoters, cell type specific promoters, temporal promoters or inducible promoters to add increasingly granular expression control of effectors and regulatory proteins. In this study we combined the E riboswitch with the strong synthetic promoter T5 and demonstrated that the riboswitch efficiently repressed translation of Clover and was strongly inducible by theophylline. In addition this induction was dose responsive providing 


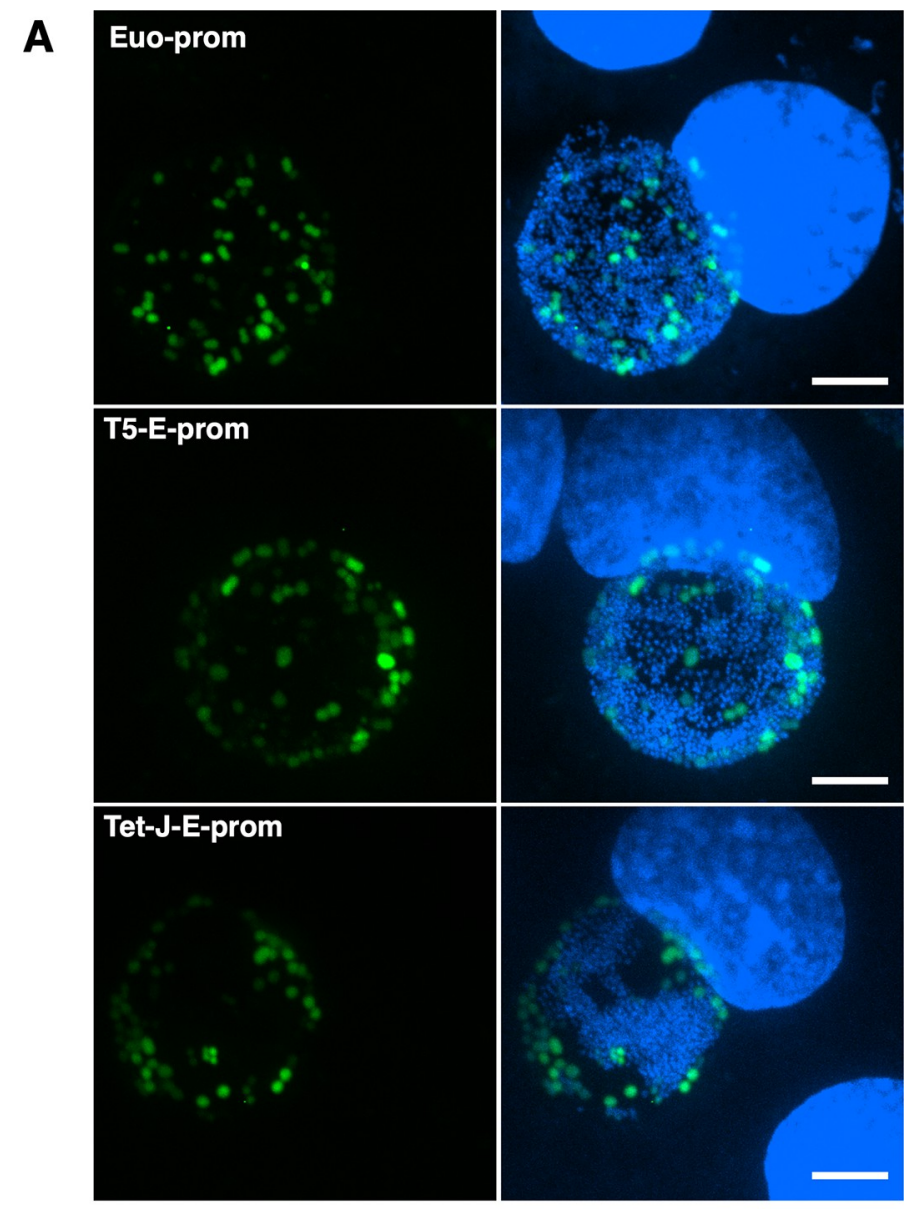

B

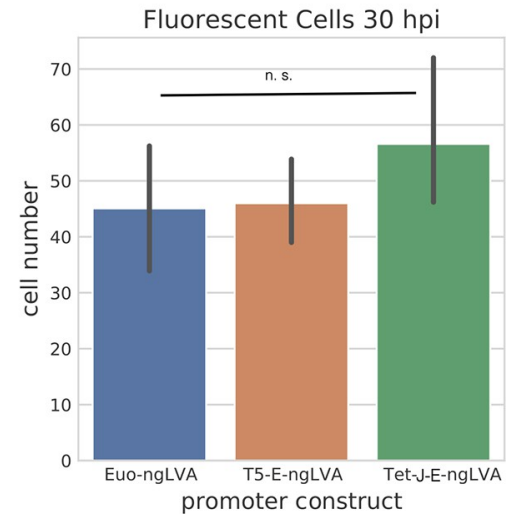

Fig 6. Promoter cell type expression. A) Confocal micrographs of Cos-7 cells infected with L2-euo-neogreenLVA (euo-ngLVA), L2-T5-E-neogreenLVA (T5-E-ngLVA), or L2-Tet-J-E-neogreenLVA (Tet-E-ngLVA) (green) and fixed and stained with DAPI (blue) at $30 \mathrm{hpi}$. Size bar $=10 \mu \mathrm{m}$. B) Quantification of $>20$ neongreenLVA expressing chlamydial cells for each promoter construct. Error bars $=$ SEM. n. s. denotes p-values $>0.05$.

https://doi.org/10.1371/journal.pone.0257259.g006

an excellent tool for the control of ectopic gene expression. We recently published the use of the T5prom-E riboswitch for the ectopic expression of three chlamydial proteins, dbdA, hctA, and sccl demonstrating its utility in dissecting the function of chlamydial proteins [17]. 
In addition to combining translational expression control with a strong promoter, we also demonstrated that it can be used with a native chlamydial promoter. The E riboswitch was cloned upstream of the ORF for the plasmid gene pgp4. Pgp4 is a gene expression regulator for both chromosomal and plasmids genes and the absence of $p g p 4$ results in a loss of glycogen accumulation in the inclusion [33]. The addition of the E riboswitch led to undetectable levels of Pgp4 expression and the loss of glycogen accumulation when theophylline was absent. The addition of theophylline during infection restored functional levels of Pgp4 as demonstrated by the restoration of glycogen accumulation in the inclusion and detectable expression of Pgp4 via western blot and confocal microscopy.

By combining translational control with transcriptional control we were able to improve the repression of protein expression. The T5 promoter-E-riboswitch combination proved to have undetectable expression when driving Clover expression as assessed by western blotting and confocal microscopy. However, when attempting to express the chlamydial protein HctB, a protein involved in controlling the developmental cycle, leaky expression resulted in mutation of the plasmid causing $\mathrm{HctB}$ to not express. It is interesting to note that ectopic expression of HctA using just the T5prom-E system transformed into chlamydia without mutations suggests low levels of HctA may be more tolerated.

By combining translational control (E riboswitch) with transcription control (Tet inducible promoter), we created an extremely tightly regulated gene expression system. The E riboswitch requires the 5'-UTR of the transcript to properly fold and block the ribosome binding site of the transcript. Combining the $\mathrm{E}$ riboswitch with different promoters and different transcription start sites can potentially affect the folding of the riboswitch, thus changing its repression and induction properties. To eliminate this effect and increase the reliability of the riboswitch in relation to a variety of promoters, we cloned the riboJ ribozyme insulator upstream of the $\mathrm{E}$ riboswitch. The riboJ insulator is made up of the sTRSV-ribozyme with an additional 23-nt hairpin immediately downstream $[35,40,41]$. This hairpin imposes structure to the UTR just upstream of the E riboswitch, minimizing its influence on the folding of the riboswitch and ensuring any upstream structure is consistent between promoters.

Surprisingly, the order of induction (transcription vs translation) did not significantly change the gene expression kinetics suggesting that there was not an accumulation of transcripts after Tet induction that then could be induced to initiate translation. Instead, this observation suggests the transcripts either don't accumulate or, after folding into the inhibited structure in the absence of theophylline they don't then refold revealing the RBS upon theophylline addition. This suggests theophylline binding competes with inhibitory folding during mRNA synthesis.

$\mathrm{HctB}$, when cloned into this dual induction plasmid was successfully transformed into Ctr and was inducible with the addition of both theophylline and aTc as detected by western blotting and confocal microscopy. Additionally, ectopic expression of HctB early in infection (16 hpi) significantly reduced the formation of infectious progeny. Together, these data confirm that leaky expression from T5-E likely rendered successful transformation of this clone impossible. Therefore, the combination of transcriptional and translational control is an ideal system to study the effects of toxic proteins or proteins that regulate the developmental cycle.

Interestingly, both the T5-E and Tet-riboJ-E promoter systems appear to only significantly express in the RB cell type. The promoters for both of these constructs are based on E. coli sigma70 consensus sequences and are constitutive in many bacteria [42, 43]. In Ctr these promoters appear to express primarily in the $\mathrm{RB}$ cell type suggesting gene expression in the intermediate body (IB) and EB cell type may require specific promoters or additional regulatory elements. Our data suggest that the ability to add translational control independently from 
transcriptional control using riboJ ribozyme and E riboswitch will be an important tool in controlling ectopic gene expression in these chlamydial cell types.

Adding inducible translational control to the tool box for chlamydial genetic tools increases opportunities to unveil the function of Ctr regulatory genes and effector genes to reveal their role in pathogenesis. The ability to control the timing and strength of gene expression independently from promoter strength and timing increases the utility of ectopic gene expression and provides an important tool for studying chlamydial pathogenesis.

\section{Supporting information}

S1 Fig. IFU results at 30 hpi. A) Cos-7 cells were infected with L2-T5-E-clover-flag and the production of infectious progeny was determined at $30 \mathrm{hpi}$ after theophylline induction or vehicle only. B) Cos-7 cells were infected with L2-nprom-E-pgp4-flag and the production of infectious progeny was determined at $30 \mathrm{hpi}$ after theophylline induction or vehicle only. C) Cos-7 cells were infected with L2-Tet-J-E-clover-flag and the production of infectious progeny was determined at $30 \mathrm{hpi}$ after induction with $0.5 \mathrm{mM}$ theophylline, $30 \mathrm{ng} / \mathrm{ml}$ aTc, both aTc and theophylline or vehicle only at $16 \mathrm{hpi}$. D) Cos-7 cells were infected with L2-Tet-J-E-hctBflag and the production of infectious progeny was determined at $30 \mathrm{hpi}$ after induction with $0.5 \mathrm{mM}$ theophylline, $30 \mathrm{ng} / \mathrm{ml}$ aTc, both aTc and theophylline or vehicle only at $16 \mathrm{hpi}$. Asterisks denote $\mathrm{p}$-values $<0.05$. Error bars $=$ SEM.

(TIF)

S2 Fig. RNA-seq analysis of p2TK2-SW2-Tetprom-riboJ-E-hctB-flag. Cos-7 cells infected with L2-Tet-J-E-hctB-flag were induced with $0.5 \mathrm{mM}$ theophylline and $30 \mathrm{ng} / \mathrm{ml}$ aTc at $15 \mathrm{hpi}$ and RNA was harvested at $24 \mathrm{hpi}$. RNA was processed for next-gen RNA-seq sequencing. Aligned reads are shown with the schematic of the Tet-J-E-hctB.

S3 Fig. ngLVA degradation kinetics. Cos-7 cell infected with L2-euoprom-ngLVA, treated with $34 \mu \mathrm{g} / \mathrm{ml}$ chloramphenicol (CAM) or vehicle only (1:1000 EtOH in RPMI) at $35 \mathrm{hpi}$ (arrow). The infections were monitored using live cell imaging for 50 hours. Expression intensities from $>50$ individual inclusions were measured via automated live-cell fluorescence microscopy and the mean intensities are shown. Cloud represents SEM. Y-axes are denoted in scientific notation. Chloramphenicol treated sample showed a decrease of half max intensity 30 mins after treatment.

(TIFF)

S4 Fig. Uncropped western blots.

(PDF)

S1 Table. Primers and templates used for plasmid construction. (PDF)

\section{Acknowledgments}

We would like to thank Dr. Paul Beare at the Rocky Mountain Labs for providing us the Tet repressor and promoter sequence. We would also like to thanks Dr. John-Demian (JD) Sauer for providing us with the T5prom-E promoter and riboswitch.

\section{Author Contributions}

Conceptualization: Nicole A. Grieshaber, Scott S. Grieshaber. 
Data curation: Scott S. Grieshaber.

Formal analysis: Nicole A. Grieshaber, Scott S. Grieshaber.

Funding acquisition: Nicole A. Grieshaber, Scott S. Grieshaber.

Investigation: Nicole A. Grieshaber, Travis J. Chiarelli, Cody R. Appa, Grace Neiswanger, Kristina Peretti, Scott S. Grieshaber.

Supervision: Nicole A. Grieshaber, Scott S. Grieshaber.

Writing - original draft: Nicole A. Grieshaber, Scott S. Grieshaber.

\section{References}

1. Owusu-Edusei KJ, Chesson HW, Gift TL, Tao G, Mahajan R, Ocfemia MCB, et al. The Estimated Direct Medical Cost of Selected Sexually Transmitted Infections in the United States, 2008. Sex Transm Dis. 2011; 40: 197201. https://doi.org/10.1097/OLQ.0b013e318285c6d2 PMID: 23403600

2. Owusu-Edusei K, Roby TM, Chesson HW, Gift TL. Productivity costs of nonviral sexually transmissible infections among patients who miss work to seek medical care: evidence from claims data. Sex Health. CSIRO PUBLISHING; 2011; 10: 434437. https://doi.org/10.1071/SH13021 PMID: 23987746

3. Torrone EA, Bernstein KT. Surveillance for Sexually Transmitted Diseases. Concepts and Methods in Infectious Disease Surveillance. John Wiley \& Sons, Ltd; 2014. pp. 122131. https://doi.org/10.1002/ 9781118928646.ch12

4. Bbar C, de Barbeyrac B. Genital Chlamydia trachomatis infections. Clin Microbiol Infect Off Publ Eur Soc Clin Microbiol Infect Dis. 15: 410. https://doi.org/10.1111/j.1469-0691.2008.02647.x PMID: 19220334

5. Ohman H, Tiitinen A, Halttunen M, Lehtinen M, Paavonen J, Surcel H-M. Cytokine polymorphisms and severity of tubal damage in women with Chlamydia-associated infertility. J Infect Dis. 199: 13531359. https://doi.org/10.1086/597620 PMID: 19358670

6. Miller WC, Ford CA, Morris M, Handcock MS, Schmitz JL, Hobbs MM, et al. Prevalence of chlamydial and gonococcal infections among young adults in the United States. JAMA: J Am Med Assoc. 291: 22292236. https://doi.org/10.1001/jama.291.18.2229 PMID: 15138245

7. Datta SD, Torrone E, Kruszon-Moran D, Berman S, Johnson R, Satterwhite CL, et al. Chlamydia trachomatis trends in the United States among persons 14 to 39 years of age, 1999-2008. Sex Transm Dis. 39: 9296. https://doi.org/10.1097/OLQ.0b013e31823e2ff7 PMID: 22249296

8. Bebear C, Barbeyrac BD. Genital Chlamydia trachomatis infections. Clinical Microbiology and Infection. Elsevier; 2009. pp. 410. https://doi.org/10.1111/j.1469-0691.2008.02647.x PMID: 19220334

9. Brothwell JA, Muramatsu MK, Zhong G, Nelson DE. Advances and Obstacles in the Genetic Dissection of Chlamydial Virulence. Current Topics in Microbiology and Immunology. Springer Nature; 2018. pp. 133158. https://doi.org/10.1007/82_2017_76 PMID: 29090367

10. Rahnama M, Fields KA. Transformation of Chlamydia: current approaches and impact on our understanding of chlamydial infection biology. Microbes and Infection. Elsevier; 2018. pp. 445450. https://doi. org/10.1016/j.micinf.2018.01.002 PMID: 29409975

11. Thomson NR, Clarke IN. Chlamydia trachomatis: small genome, big challenges. Future Microbiology. Future Medicine; 2010. pp. 55561. https://doi.org/10.2217/fmb.10.31 PMID: 20353297

12. Breaker RR. Prospects for riboswitch discovery and analysis. Molecular Cell. Elsevier; 2011. pp. 86779. https://doi.org/10.1016/j.molcel.2011.08.024 PMID: 21925376

13. Lynch SA, Gallivan JP. A flow cytometry-based screen for synthetic riboswitches. Nucleic Acids Research. Oxford University Press; 2008. pp. 18492. https://doi.org/10.1093/nar/gkn924 PMID: 19033367

14. Howard L, Orenstein NS, King NW. Purification on renografin density gradients of Chlamydia trachomatis grown in the yolk sac of eggs. Appl Microbiol. 1971; 27: 102106.

15. Agaisse $\mathrm{H}$, Derr I. A C. trachomatis cloning vector and the generation of $\mathrm{C}$. trachomatis strains expressing fluorescent proteins under the control of a $\mathrm{C}$. trachomatis promoter. PLoS ONE. Public Library of Science; 2013. https://doi.org/10.1371/JOURNAL.PONE.0057090 PMID: 23441233

16. Vlack ERV, Seeliger JC. Using riboswitches to regulate gene expression and define gene function in mycobacteria. Methods in Enzymology. Elsevier; 2014. pp. 25165. https://doi.org/10.1016/bs.mie. 2014.10.034 PMID: 25605389 
17. Grieshaber NA, Runac J, Turner S, Dean M, Appa C, Omsland A, et al. The sRNA Regulated Protein DdbA Is Involved in Development and Maintenance of the Chlamydia trachomatis EB Cell Form. Frontiers in Cellular and Infection Microbiology. Frontiers Media S.A.; 2021. https://doi.org/10.3389/fcimb. 2021.692224 PMID: 34368013

18. Tinevez J-Y, Perry N, Schindelin J, Hoopes GM, Reynolds GD, Laplantine E, et al. TrackMate: An open and extensible platform for single-particle tracking. Methods. Academic Press; 2015; 115: 8090. https:// doi.org/10.1016/j.ymeth.2016.09.016 PMID: 27713081

19. Chiarelli TJ, Grieshaber NA, Omsland A, Remien CH, Grieshaber SS. Single Inclusion Kinetics of Chlamydia trachomatis Development. mSystems.00689-20. mSystems. 2020. https://doi.org/10.1128/ mSystems.00689-20 PMID: 33051378

20. Langdon WB. Performance of genetic programming optimised Bowtie2 on genome comparison and analytic testing (GCAT) benchmarks. BioData Min. Department of Computer ScienceUniversity College London, Gower Street, London, WC1E 6BT UK.: BioMed Central; 2013; 8: 1. https://doi.org/10.1186/ s13040-014-0034-0 PMID: 25621011

21. Anders S, Pyl PT, Huber W. HTSeq-a Python framework to work with high-throughput sequencing data. Bioinforma. 31: 166169. https://doi.org/10.1093/bioinformatics/btu638 PMID: 25260700

22. Love MI, Huber $\mathrm{W}$, Anders $\mathrm{S}$. Moderated estimation of fold change and dispersion for RNA-seq data with DESeq2. Genome Biol. BioMed Central; 2012; 15: 550. https://doi.org/10.1186/s13059-014-05508 PMID: 25516281

23. Haslwanter T. An Introduction to Statistics with Python: With Applications in the Life Sciences 2013. Available: $\mathrm{http}: / /$ books. google.com/books? $\mathrm{hl}=$ en\&lr=\&id=ZBi1DAAAQBAJ\&oi=fnd\&pg=PR7\&dq= seaborn python\&ots=47D22VZUbM\&sig=GPJ6-XGP0EjLwWnQI17BI_fp0SY. https://doi.org/10.1002/ eji.201343681 PMID: 24165969

24. Rudolph MM, Vockenhuber MP, Suess B. Conditional control of gene expression by synthetic riboswitches in Streptomyces coelicolor. Methods in Enzymology. Elsevier; 2014. pp. 28399. https://doi.org/ 10.1016/bs.mie.2014.10.036 PMID: 25605391

25. Topp S, Reynoso CMK, Seeliger JC, Goldlust IS, Desai SK, Murat D, et al. Synthetic riboswitches that induce gene expression in diverse bacterial species. Appl Environ Microbiol. Department of Chemistry, Center for Fundamental and Applied Molecular Evolution, Emory University, Atlanta, GA 30322, USA.: American Society for Microbiology; 2008; 76: 78817884. https://doi.org/10.1128/AEM.01537-10 PMID: 20935124

26. Bryksin AV, Matsumura I. Rational design of a plasmid origin that replicates efficiently in both gram-positive and gram-negative bacteria. PLoS ONE. Public Library of Science; 2010. https://doi.org/10.1371/ journal.pone.0013244 PMID: 20949038

27. Gentz R, Bujard H. Promoters recognized by Escherichia coli RNA polymerase selected by function: highly efficient promoters from bacteriophage T5. Journal of Bacteriology. American Society for Microbiology; 1985. pp. 707. https://doi.org/10.1128/jb.164.1.70-77.1985 PMID: 3900050

28. Cui W, Han L, Cheng J, Liu Z, Zhou L, Guo J, et al. Engineering an inducible gene expression system for Bacillus subtilis from a strong constitutive promoter and a theophylline-activated synthetic riboswitch. Microbial Cell Factories. Springer Nature; 2016. https://doi.org/10.1186/s12934-016-0599-z PMID: 27876054

29. Wang Y, Kahane S, Cutcliffe LT, Skilton RJ, Lambden PR, Clarke IN. Development of a Transformation System for Chlamydia trachomatis: Restoration of Glycogen Biosynthesis by Acquisition of a Plasmid Shuttle Vector. PLoS Pathog. Public Library of Science; 2011; 7: 1002258. Available: http://dx.plos.org/ 10.1371/journal.ppat.1002258. https://doi.org/10.1371/journal.ppat.1002258 PMID: 21966270

30. Chiarelli TJ, Grieshaber NA, Grieshaber SS. Live-Cell Forward Genetic Approach to Identify and Isolate Developmental Mutants in Chlamydia trachomatis JoVE. MyJoVE Corp; 2020. p. e61365. https://doi. org/10.3791/61365 PMID: 32597859

31. Zhang Q, Rosario CJ, Sheehan LM, Rizvi SM, Brothwell JA, He C, et al. The Repressor Function of the Chlamydia Late Regulator EUO Is Enhanced by the Plasmid-Encoded Protein Pgp4. Journal of Bacteriology. American Society for Microbiology; 2020. https://doi.org/10.1128/JB.00793-19 PMID: 31988079

32. Gong S, Yang Z, Lei L, Shen L, Zhong G. Characterization of Chlamydia trachomatis Plasmid-Encoded Open Reading Frames. J Bacteriol. 195: 38193826. https://doi.org/10.1128/JB.00511-13 PMID: 23794619

33. Song L, Carlson JH, Whitmire WM, Kari L, Virtaneva K, Sturdevant DE, et al. Chlamydia trachomatis plasmid-encoded Pgp4 is a transcriptional regulator of virulence-associated genes. Infect Immun. 81: 636644. https://doi.org/10.1128/IAI.01305-12 PMID: 23319558

34. Beare PA, Gilk SD, Larson CL, Hill J, Stead CM, Omsland A, et al. Dot/lcm type IVB secretion system requirements for Coxiella burnetii growth in human macrophages. MBio. American Society for Microbiology; 2011. pp. e00175-11. https://doi.org/10.1128/mBio.00175-11 PMID: 21862628 
35. Clifton KP, Jones EM, Paudel S, Marken JP, Monette CE, Halleran AD, et al. The genetic insulator RiboJ increases expression of insulated genes. Journal of Biological Engineering. Springer Nature; 2018. https://doi.org/10.1186/s13036-018-0115-6 PMID: 30386425

36. Grieshaber NA, Tattersall JS, Liguori J, Lipat JN, Runac J, Grieshaber SS. Identification of the basepairing requirements for repression of hctA translation by the small RNA IhtA leads to the discovery of a new mRNA target in Chlamydia trachomatis. PloS one. Department of Biological Sciences, College of Science, University of Idaho, Moscow, Idaho, United States of America.: Public Library of Science; 2013; 10: e0116593. https://doi.org/10.1371/journal.pone.0116593 PMID: 25756658

37. Lease RA. Riboregulation in Escherichia coli: DsrA RNA acts by RNA:RNA interactions at multiple loci. Proc Natl Acad Sci. 1996; 95: 1245612461. https://doi.org/10.1073/pnas.95.21.12456 PMID: 9770507

38. Reynoso CMK, Miller MA, Bina JE, Gallivan JP, Weiss DS. Riboswitches for intracellular study of genes involved in Francisella pathogenesis. mBio. Department of Chemistry, Emory University, Atlanta, Georgia, USA.: American Society for Microbiology; 2010; 3: e00253-12. https://doi.org/10.1128/mBio. 00253-12 PMID: 23169998

39. Nakahira $\mathrm{Y}$, Ogawa A, Asano H, Oyama T, Tozawa $Y$. Theophylline-dependent riboswitch as a novel genetic tool for strict regulation of protein expression in Cyanobacterium Synechococcus elongatus PCC 7942. Plant \& cell Physiol. 54: 17241735. https://doi.org/10.1093/pcp/pct115 PMID: 23969558

40. Richardson MW, Hostalek L, Dobson M, Hu J, Shippy R, Siwkowski A, et al. Design, targeting, and initial screening of sTRSV-derived hairpin ribozymes for optimum helix 1 length and catalytic efficiency in vitro. Methods in Molecular Biology (Clifton, N.J.). Springer Nature; 2004. pp. 33958. https://doi.org/10. 1385/1-59259-746-7:339.

41. Nelson JA, Shepotinovskaya I, Uhlenbeck OC. Hammerheads derived from sTRSV show enhanced cleavage and ligation rate constants. Biochemistry. American Chemical Society (ACS); 2005. pp. 1457785. https://doi.org/10.1021/bi051130t PMID: 16262257

42. Deuschle U, Kammerer W, Gentz R, Bujard H. Promoters of Escherichia coli: a hierarchy of in vivo strength indicates alternate structures. The EMBO Journal. EMBO; 1986. pp. 298794. https://doi.org/ 10.1002/J.1460-2075.1986.TB04596.X PMID: 3539589

43. Larson CL, Martinez E, Beare PA, Jeffrey B, Heinzen RA, Bonazzi M. Right on Q: genetics begin to unravel Coxiella burnetii host cell interactions. Future Microbiology. Future Medicine; 2016. pp. 91939. https://doi.org/10.2217/fmb-2016-0044 PMID: 27418426 